\title{
Chirped Lidar Using Simplified Homodyne Detection
}

\author{
Peter Adany, Christopher Allen, and Rongqing Hui
}

\begin{abstract}
A simplified homodyne detection scheme for linear FM modulated lidar is presented in which pulse dechirping is performed in the optical domain. This method provides quantum limited detection sensitivity with much less receiver complexity compared to heterodyne detection systems. Another advantage of this approach is the reduced bandwidth requirement for the photodetector. This removes the limit on the chirp bandwidth, and enables the use of more efficient photodiodes with larger detector area. A field trial using a 5-in aperture diameter commercial telescope and a 370-m target range verified the sensitivity estimation and demonstrated the feasibility of this technique.
\end{abstract}

Index Terms-Coherent optical effects, fiber optics sensors, laser range finder, lidar, optical sensing and sensors.

\section{INTRODUCTION}

$\mathbf{L}$ IDAR altimeters can provide finer range resolution and smaller beam size than conventional microwave radar systems. The range accuracy of a lidar system depends on signal bandwidth and the receiver signal-to-noise ratio (SNR). To achieve acceptable range accuracy and detection sensitivity, many long range lidar systems use short pulse lasers with low pulse repetition rate and extremely high pulse peak power. In these systems, photon damage has been a concern because peak power in the megawatt range gradually degrades the optics, shortening the lifetime of the system. The use of lower peak power affords longer lifetime as well as decreased safety hazards.

To attain acceptable sensitivity and resolution using available $\mathrm{CW}$ lasers, chirped lidar systems using long optical pulses with relatively low peak optical power have been developed [1]. A linear frequency RF chirp is used to modulate the optical pulses, and a simple de-chirping operation (mixing) converts the time delay information of return pulses into frequency readings. In this configuration, the range accuracy depends on the RF chirp bandwidth rather than the pulse duration, and therefore excellent range accuracy can still be obtained using long optical pulses with low peak power.

In the linear FM lidar, a linear frequency RF chirp is used to modulate the optical pulses, and a simple de-chirping operation (mixing) converts the time delay information of return pulses into frequency readings. In this configuration, the range accuracy depends on the RF chirp bandwidth rather than the pulse duration, and therefore excellent range accuracy can still be obtained using long optical pulses with low peak power.

Manuscript received December 08, 2008; revised February 16, 2009. Current version published July 24, 2009.

The authors are with the Department of Electrical Engineering and Computer Science, University of Kansas, Lawrence, KS 66045 USA (e-mail: peter@ittc.ku.edu; callen@eecs.ku.edu; hui@eecs.ku.edu; hui@ittc.ku.edu).

Digital Object Identifier 10.1109/JLT.2009.2016220
Under direct detection, thermal noise is the dominant noise source and the sensitivity is relatively poor. Much higher detection sensitivity can be achieved using coherent optical mixing, which can theoretically provide quantum-limited SNR. This ability has been demonstrated in a lidar system using a heterodyne architecture [1]. However, the requirements of high intermediate frequency (IF) and the subsequent RF down conversion increase the system complexity and also introduce excess losses that worsen the sensitivity.

In this paper, we present a linear FM (chirped) lidar system using a simplified homodyne detection scheme, in which the linear RF down conversion occurs in the optical domain. This method is distinct from other optical homodyne systems such as [2] because it does not rely on ramping of the laser wavelength. Instead, the transmitted pulse is intensity modulated by a linear FM chirp, and dechirping is performed by coherent mixing with a modulated local oscillator (LO). In this arrangement, the mixed signal entering the photodetector has much lower frequency bounds than the transmitted pulse, thus relaxing the photodetector bandwidth requirement. The RF receiver hardware also becomes very simple and thus makes this technique practical and easy to implement.

\section{Pulse Compression In ChIRPed LidAR With DireCt AND COHERENT HETERODYNE DETECTION}

In conventional pulsed lidar systems, the range resolution is approximately $\Delta R=c \tau / 2$, where $\tau$ is the pulse duration and $c$ is the speed of light. Clearly, fine range resolution requires short pulsewidth, which usually demands high peak power for adequate SNR. Fundamentally, range accuracy is approximately

$$
\sigma_{R}=K \frac{c}{B \sqrt{\mathrm{SNR}}}
$$

where $B$ is the signal bandwidth, SNR is the receiver signal to noise ratio and $K$ is a proportionality factor depending on the chirp waveform [3]. Equation (1) also indicates that linear FM modulation with the same bandwidth as the pulsed optical signal can provide comparable range resolution.

Linear FM chirp refers to a ramped-frequency waveform used to modulate the laser intensity. The principle is illustrated in Fig. 1, in which an RF signal frequency increases linearly from $f_{1}$ to $f_{2}$ over pulse duration $\tau$. This chirp waveform modulates the CW laser pulse which is then transmitted via a telescope. The target range information is contained in the difference frequency $f_{R}$ given by $f_{R}=\left(f_{2}-f_{1}\right) \Delta t / \tau=2 B R /(c \tau)$, where $B=\left(f_{2}-f_{1}\right)$ is the chirp bandwidth. The range information is found easily by a time domain mixing operation referred to as de-chirping.

In a chirped lidar system with direct detection as shown in Fig. 2, a chirp RF waveform is split into two parts: one part is used to drive an electro-optic intensity modulator and the other 


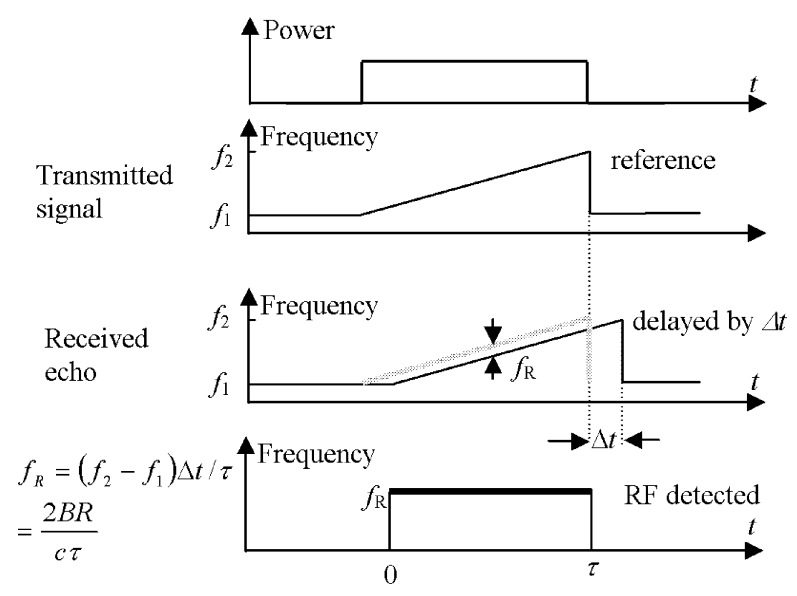

Fig. 1. Illustration of linear FM dechirping.

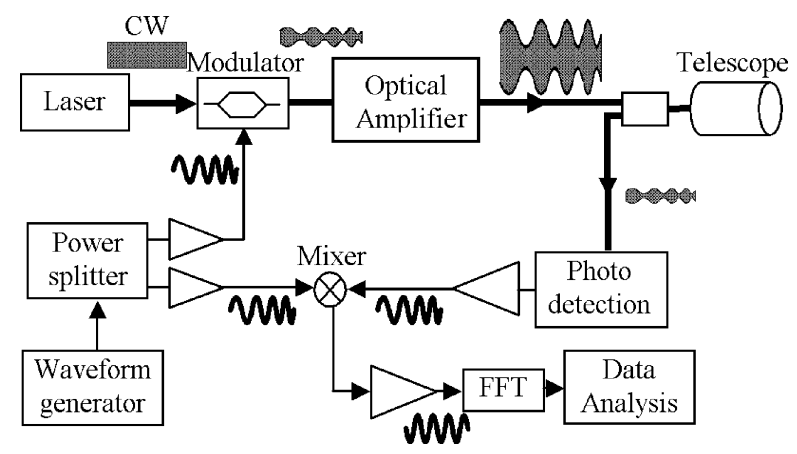

Fig. 2. Block diagram for direct detection.

part is used as the RF local oscillator for de-chirping. The modulated optical signal is amplified by an optical amplifier and sent to a telescope. The backscattered signal from the target is collected by the same telescope and detected by a photodiode, which converts the chirped optical signal back into RF domain. This recovered RF signal is amplified and beats with the RF local oscillator in a mixer where de-chirping is performed. Then the de-chirped signal is frequency-analyzed by a fast Fourier transform (FFT) process to find the beat frequency $f_{R}$ and the corresponding target range information. Since the optical signal backscattered from the target is usually very weak, the SNR at the photodiode output is predominantly limited by thermal noise. Although direct detection provides simple system architecture, the major limitation is its poor receiver sensitivity. Only considering the effect of thermal noise, the maximum theoretical SNR is [5]

$$
\mathrm{SNR}_{\mathrm{dir}} \approx \frac{2 \Re^{2} P_{\mathrm{sig}}^{2}}{\frac{4 k T B_{e}}{R_{L}}}
$$

where $\Re$ is the photodiode responsivity, $P_{\text {sig }}$ is the received signal optical power, $k$ is Planck's constant, $T$ is the absolute temperature, $B_{e}$ is the electrical bandwidth, and $R_{L}$ is the load resistance. Equation (2) shows that receiver SNR is degraded by $2 \mathrm{~dB}$ for each $\mathrm{dB}$ reduction of the received signal optical power. This is especially disadvantageous for a long-range lidar where the received optical signal level is extremely low.

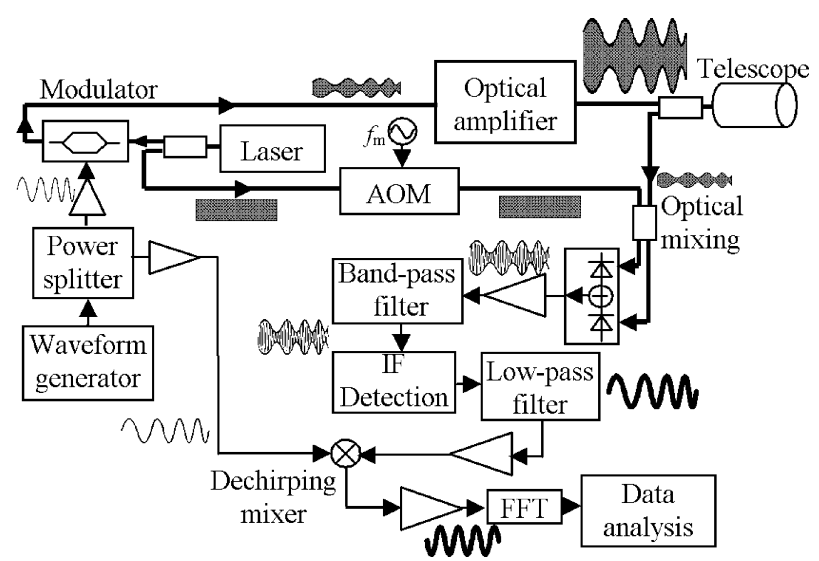

Fig. 3. Block diagram for heterodyne detection.

Coherent heterodyne detection as illustrated in Fig. 3 increases the receiver sensitivity in FM lidar systems [4]. In this system, the source laser is split into two parts: one is modulated by the RF chirp through an electro-optic intensity modulator and fed to the telescope, while the other is used as the optical local oscillator (LO). An acousto-optic modulator (AOM) is used to shift the optical frequency of LO by $f_{\mathrm{m}}$ which serves as the IF frequency for coherent heterodyne detection. In the receiver, a balanced photodiode rejects the direct detection component. The heterodyne IF signal is selected by a bandpass filter and detected by an IF envelope detector made of a Schottky diode, which recovers the baseband chirp waveform. Then a de-chirping mixer compares this detected signal with the original chirp waveform and an FFT process is used to find the beat frequency $f_{R}$. In coherent detection, because of the existence of the strong optical LO, the SNR is predominately determined by the shot noise, and the maximum theoretical SNR for coherent receiver can be evaluated as

$$
\mathrm{SNR}_{c o h} \approx \frac{\Re P_{\text {sig }}}{2 q B_{e}} .
$$

where $q$ is the electron charge. In contrast to direct detection, SNR in coherent detection is linearly proportional to the signal optical power, which is more suitable for low signal power operation. However, this coherent heterodyne system is much more complex than the direct detection. For heterodyne detection, the IF frequency is usually set at a few $\mathrm{GHz}$ in order to be much higher than the baseband, and this requires high speed optical detection and RF processing circuitry. Most importantly, there is an intrinsic signal processing loss due to the mixing between the signal and the RF noise in the IF envelope detection process, which may significantly degrade SNR. Therefore quantum noise limited SNR predicted by (3) has not been achieved using coherent heterodyne detection [1].

\section{FM LidAR With Simplified Homodyne Detection}

\section{A. System Description}

To simplify coherent detection while maintaining its advantage of high receiver sensitivity, we propose a modified homodyne detection scheme, which simultaneously performs fre- 


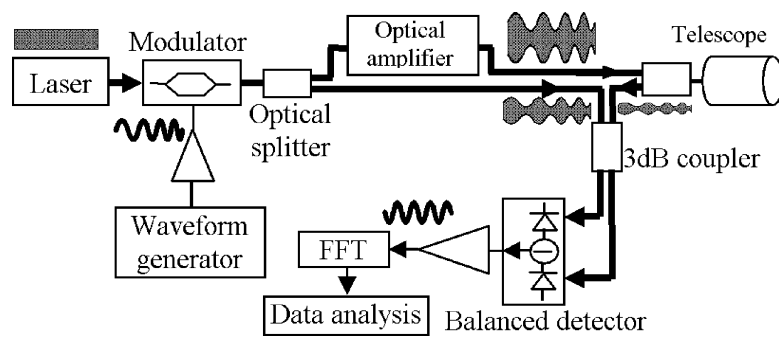

Fig. 4. Block diagram for homodyne self-chirped detection.

quency down-conversion and de-chirping in the photodetector. As shown in Fig. 4, in this system the optical signal from a CW laser is modulated by a chirp waveform through an electro-optic modulator. This intensity modulated optical signal is then split into two parts. One part is amplified and sent to the telescope, while the other part is used as the LO. The backscattered optical signal picked up by the telescope is combined with the LO through a $2 \times 2$ optical coupler and detected by a balanced photodetector. The key difference between this simplified homodyne detection and a conventional homodyne detection is that in this system both the transmitted signal and the LO are modulated by the same RF chirp waveform. The optical mixing associated with coherent detection in the photodiode simultaneously performs optical detection and RF de-chirping, and therefore additional RF signal mixing de-chirping are no longer necessary and the system becomes greatly simplified. A frequency analysis of the detected photocurrent using FFT directly produces the range information of the target.

Based on the principle of coherent homodyne detection, if the 3-dB optical coupler is ideal, the signal component of the photocurrent obtained at the output of the photodetector is

$$
\begin{aligned}
I_{\mathrm{sig}}=2 \Re \sqrt{2} E_{L O} \cos (\Omega(t) \cdot t) E_{\mathrm{sig}} \\
\cdot \cos (\Omega(t-\Delta t) \cdot t) \sin (\delta \phi(t))
\end{aligned}
$$

where $E_{L O} \cos (\Omega(t) \cdot t)$ is the amplitude of the chirped LO and $E_{\text {sig }} \cos (\Omega(t-\Delta t) \cdot t)$ is the detected signal amplitude which is a delayed version of the $\mathrm{LO}$. The direct detection component is not present in (4) because it is eliminated in the differential photo current from the balanced photodiodes. The term

$$
\Omega(t)=f_{1}+\frac{\left(f_{2}-f_{1}\right) t}{\tau}
$$

is the linear FM chirp which is intensity modulated on the optical carrier. $\tau$ is the optical pulse duration within which the modulation frequency is linearly changed from $f_{1}$ to $f_{2}$. $\Delta t$ is the roundtrip propagation delay between the telescope and the target. $\delta \phi(\mathrm{t})$ is the optical phase mismatch between the received optical signal and the LO, which may fluctuate over time due to laser phase noise as well as air turbulence between the telescope and the target. De-chirping takes place in the photodiode due to the mixing between the local oscillator and its time delayed copy in the coherent detection process. The frequency difference between the $\mathrm{LO}$ and the received optical signal can be determined by a Fourier analysis of the photocurrent signal given by (4) as

$$
f_{R}=\Omega(t)-\Omega(t-\Delta t)=\left(\frac{f_{2}-f_{1}}{\tau}\right) \Delta t
$$

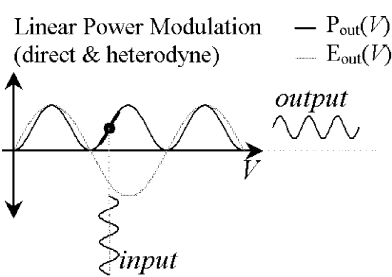

(a)

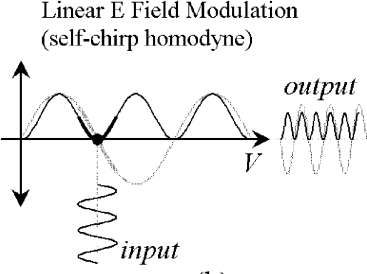

(b)
Fig. 5. Illustration of the EOM transfer function for $E$ field and power.

Therefore, the target distance can be easily calculated by $R=$ $c \Delta t / 2=c f_{R} \tau / 2\left(f_{2}-f_{1}\right)$.

\section{B. Optimum Biasing of Electro-Optic Modulator}

It is important to note that in a coherent receiver the optical phase information is preserved, which allows the use of a modulation format similar to the duobinary modulation commonly used in optical communication systems [7]. The system can be implemented by using a Mach-Zehnder electro-optic intensity modulator, whose $\mathrm{E}$ field transfer function can be expressed as

$$
T(V)=\frac{E_{\text {out }}}{E_{\text {in }}}=\cos \left(\phi_{0}+\frac{\pi V}{2 V_{\pi}}\right)
$$

where the transmission loss is neglected. $E_{\text {in }}$ and $E_{\text {out }}$ are the input and output optical fields, respectively, $V_{\pi}$ is the voltage required to change the output optical power from the minimum to the maximum [5] and $\phi_{0}$ is an initial phase which depends on the bias condition. In intensity detection-based optical systems, the modulator is most often biased at the quadrature point with $\phi_{0}= \pm \pi / 4$ to ensure the maximum output power change with the swing of signal electrical voltage $V_{\text {in }}$ between $\pm V_{\pi} / 2$, as illustrated in Fig. 5(a). In a coherent system, on the other hand, the optimum bias should be at the null point of the power transfer function where the field transmission $T(V)$ has the highest slope. This doubles the modulation frequency and increases the modulation index, and also guarantees that the quiescent optical power output is zero when there is no RF modulation, as shown in Fig. 5(b). This acts like a time gate to shut off the LO between pulses, limiting the unnecessary shot noise contribution in the coherent receiver. This optimization of modulator biasing is experimentally verified as discussed in the next section.

\section{System SNR Testing and Comparison}

To validate our simplified homodyne receiver concept and compare its performance with other lidar detection schemes, we assembled experimental lidar systems with direct detection, coherent heterodyne detection and the simplified homodyne detection. A diode pumped 1319-nm Nd:YAG laser was used as the source. The FM chirp was generated by an arbitrary waveform generator which is used to drive an electro-optic Mach-Zehnder modulator. The optical pulse duration was $\tau=40 \mu \mathrm{s}$ and the pulse repetition rate was $9.4 \mathrm{kHz}(\sim 106 \mu \mathrm{s}$ pulse separation), yielding a duty cycle of approximately $38 \%$. The modulation frequency was linearly chirped from $f_{1}=100 \mathrm{MHz}$ to $f_{2}=300 \mathrm{MHz}$ within each pulse, producing a $5-\mathrm{MHz} / \mu$ s chirp rate. A balanced photodiode with an 


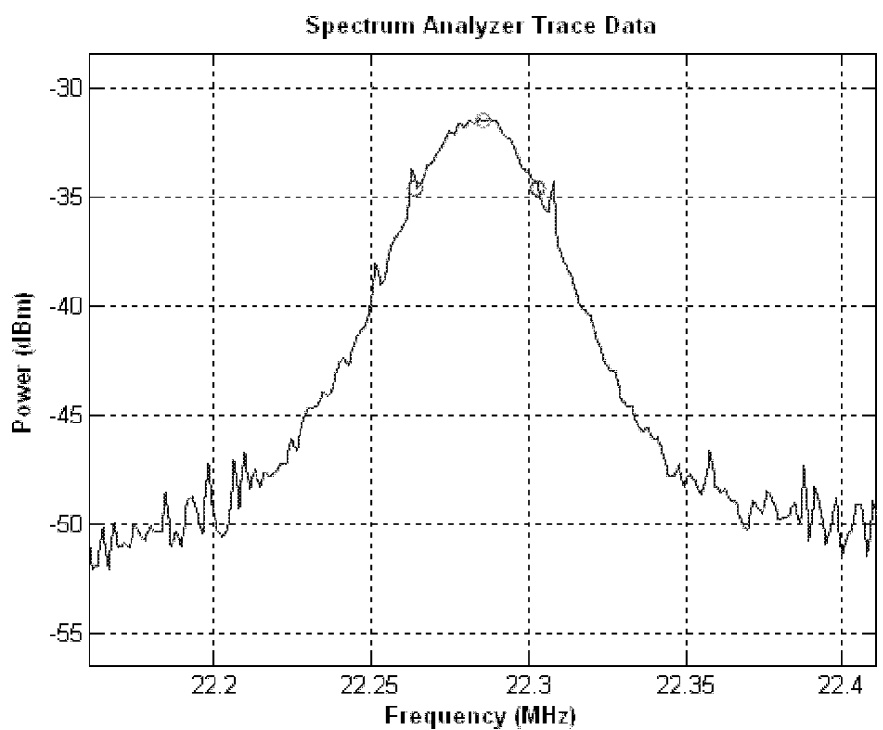

Fig. 6. Example of the measured de-chirped beat signal.

$800-\mathrm{MHz}$ bandwidth was used as the detector for both direct and coherent homodyne detections. Since coherent heterodyne detection requires a much wider receiver bandwidth to accommodate the IF frequency, a photodiode with a $20-\mathrm{GHz}$ bandwidth was used with the IF set at $15 \mathrm{GHz}$. In order to find $f_{R}$, an RF spectrum analyzer was used to perform signal analysis and the resolution bandwidth was set to $30 \mathrm{kHz}$. To avoid the uncertainties due to target reflectivity, the coupling efficiency of the telescope and turbulence of free space transmission, a $22.7-\mathrm{km}$ standard single-mode optical fiber was used to introduce a propagation delay of $110.4 \mu$ s. Fig. 6 shows an example of the de-chirped beat frequency produced by the simplified homodyne system. The de-chirped frequency $f_{R}$ at approximately $22 \mathrm{MHz}$ is determined by the difference between the propagation delay of $110.4 \mu$ s and the delay of $106 \mu$ s between successive optical pulses, as

$$
f_{R}=\frac{200 \mathrm{MHz}}{40 \mu \mathrm{s}}(110.4 \mu \mathrm{s}-106 \mu \mathrm{s})=22 \mathrm{MHz} .
$$

The spectral linewidth of the de-chirped RF signal is approximately $39 \mathrm{kHz}$, which is determined by the chirping bandwidth $B$. The SNR for each of the three systems was evaluated by recording the de-chirped signal trace data from an RF spectrum analyzer and simultaneously measuring the downconverted signal power and the noise power spectral density. Fig. 7 shows the measured SNR versus received optical power for direct detection, coherent heterodyne detection and simplified coherent homodyne detection. The theoretical shot-noise limited SNR predicted by (3) is marked by the dashed line in the same figure for comparison.

As expected, the slope of the measured SNR versus the received signal optical power is approximately $2 \mathrm{~dB} / \mathrm{dB}$ for direct detection and $1 \mathrm{~dB} / \mathrm{dB}$ for coherent detection. For the simplified homodyne detection, a maximum sensitivity of $-96 \mathrm{dBm}$ was obtained for 10-dB SNR, which approaches the theoretical quantum limit given by (3). This detection sensitivity is approximately $30 \mathrm{~dB}$ better compared to the heterodyne detection in the same system. The sensitivity degradation of heterodyne de-

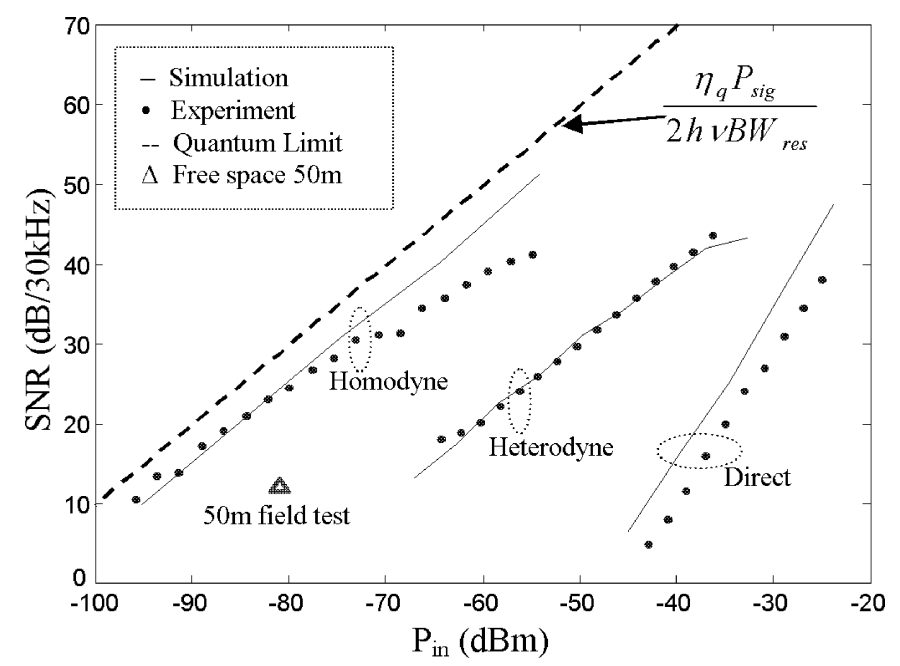

Fig. 7. Discrete dots: measured SNR of direct, heterodyne and self chirped homodyne detection with $30-\mathrm{kHz}$ spectrum analyzer resolution bandwidth. No averaging is used. Solid lines: calculated SNR values using numerical simulation. Dashed line: quantum limit. Triangle: result of field test through a 5-in telescope.

tection could be partly explained by the losses arising from IF down conversion (envelope detection) and RF de-chirping. In the IF envelope detection process, the signal mixes with the RF noise which effectively amplifies the noise contribution and degrades the SNR [8]. In addition, because heterodyne detection requires a higher IF frequency, a wide bandwidth photodetector has to be used. In general, wide bandwidth photodiodes have lower responsivity. In our experiment the responsitivity of the high speed photodetector used in heterodyne detection is about $69 \%$ of the lower speed photodetector used for homodyne detection, which accounts for about 3.25-dB SNR degradation. Solid lines in Fig. 7 were obtained from numerical simulations.

To investigate the impact of modulator biasing on the performance of the simplified coherent homodyne lidar system, we measured the SNR as a function of the modulator bias voltage. Fig. 8 shows the measured SNR and the normalized modulator power transfer function (S21) versus the modulator bias voltage. It is evident that the highest SNR was obtained at the bias point where the modulator power transfer function $\mathrm{S}_{21}$ is at its minimum. If the modulator is biased at the quadrature point, there will be an approximately $3-\mathrm{dB}$ SNR degradation compared to the optimum biasing.

\section{Phase Diversity Receiver}

In the absence of Doppler shift in the received pulse wavelength, the homodyne system is vulnerable to carrier fading. In the coherent mixing operation, the relative phase fluctuates naturally due to laser phase noise as well as mechanical vibrations and air turbulence. This is represented as a random fluctuation of $\delta \phi(t)$ in (4). In fact, we have observed significant random fading of the beat signal in our experiments due to optical phase fluctuations. To overcome this problem, a phase-diversity receiver can be implemented by inserting a unique $90^{\circ}$-hybrid optical coupler in place of the conventional 3-dB coupler before the balanced photo-detector. The two photodiode output signals are measured separately and their powers are added together. 


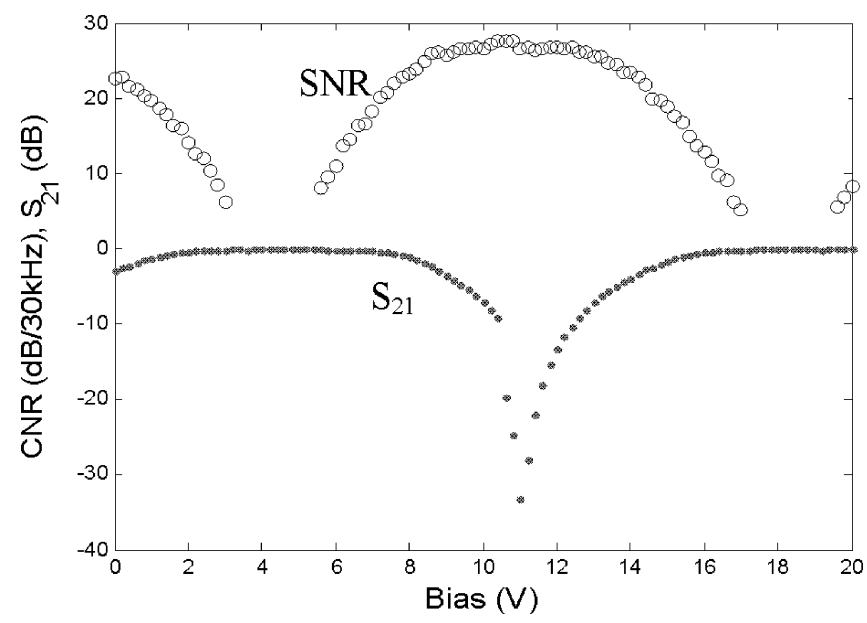

Fig. 8. SNR and EOM DC transmission coefficient versus bias voltage.

In coherent receivers with balanced detection a $2 \times 2$ fiber directional coupler is usually used. A conventional $2 \times 2$ fiber coupler is considered a $180^{\circ}$ optical hybrid, and the optical phase contributions have opposite signs in the photocurrents of the two detectors. Therefore, the subtraction of these two photocurrents removes the direct detection components while doubling the coherent detection efficiency.

In a phase diversity receiver, a $90^{\circ}$-hybrid optical coupler is a key component. While there are a number of ways to implement the $90^{\circ}$-hybrid, we used a $3 \times 3$ fiber coupler in our experiment with its scattering matrix described by [6]

$$
\begin{aligned}
& {\left[\begin{array}{l}
E_{01} \\
E_{02} \\
E_{03}
\end{array}\right]} \\
& =\left(\begin{array}{ccc}
\sqrt{0.2} & \sqrt{0.4} \exp \left(j \frac{3 \pi}{4}\right) & \sqrt{0.4} \exp \left(j \frac{3 \pi}{4}\right) \\
\sqrt{0.4} \exp \left(j \frac{3 \pi}{4}\right) & \sqrt{0.2} & \sqrt{0.4} \exp \left(j \frac{3 \pi}{4}\right) \\
\sqrt{0.4} \exp \left(j \frac{3 \pi}{4}\right) & \sqrt{0.4} \exp \left(j \frac{3 \pi}{4}\right) & \sqrt{0.2}
\end{array}\right) \\
& \cdot\left[\begin{array}{l}
E_{i 1} \\
E_{i 2} \\
E_{i 3}
\end{array}\right] .
\end{aligned}
$$

where $E_{\mathrm{ik}}$ and $E_{0 k}(k=1,2,3)$ are the input and the output optical fields, respectively, at various fiber ports as illustrated in Fig. 9. If the input ports 1 and 2 are connected to the received optical signal and the LO, respectively, and the output ports 1 and 2 are connected to the two balanced photodiodes, the two photocurrents are

$$
\begin{aligned}
I_{\mathrm{arm} 1}= & \Re\left|E_{01}\right|^{2}=\Re \sqrt{0.32} E_{L O} \cos (\Omega(t) \cdot t) E_{\mathrm{sig}} \\
& \cdot \cos (\Omega(t-\Delta t) \cdot t) \cos \left(\delta \phi(t)-\frac{3 \pi}{4}\right) \\
I_{\mathrm{arm} 2}= & \Re\left|E_{02}\right|^{2}=\Re \sqrt{0.32} E_{L O} \cos (\Omega(t) \cdot t) E_{\mathrm{sig}} \\
& \cdot \cos (\Omega(t-\Delta t) \cdot t) \cos \left(\delta \phi(t)+\frac{3 \pi}{4}\right) .
\end{aligned}
$$

The random fading represented by the last term in $(9 a)$ and (9b) become complementary due to the $\pm 3 / 4 \pi$ phase offset. Although the RF output from both photodiodes $I_{\text {arm } 1}$ and $I_{\text {arm } 2}$

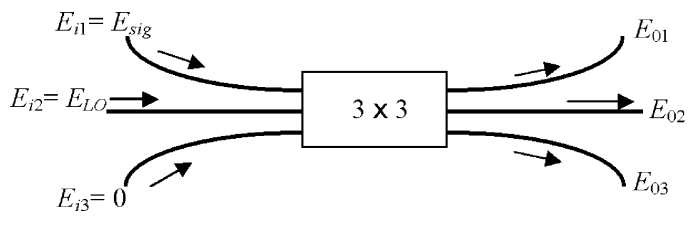

Fig. 9. Illustration of a $3 \times 3$ fiber optical coupler for phase-diversity receiver.

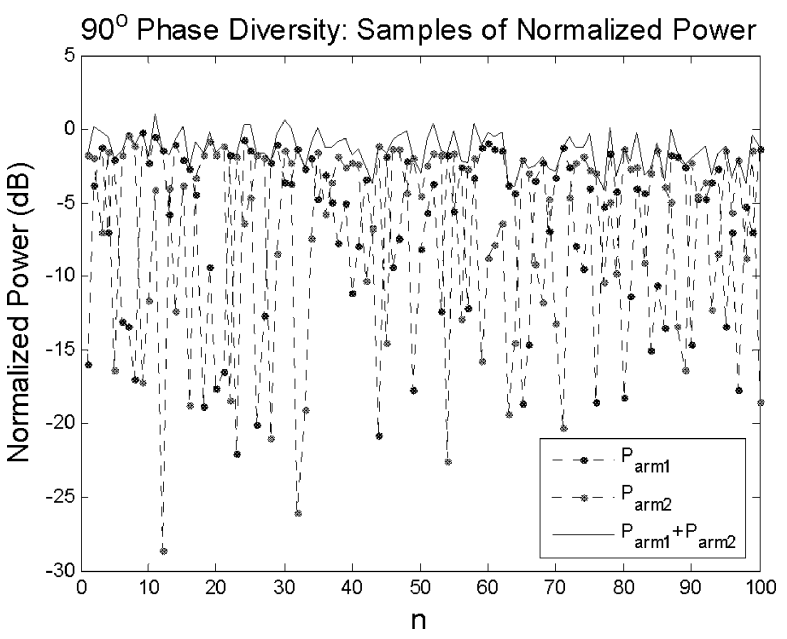

Fig. 10. Samples of normalized received power, individually and summed.

are functions of $\delta \phi(t)$, the combined RF power of the two photodiode outputs, $P_{R F}=I_{\mathrm{arm} 1}^{2}+I_{\mathrm{arm} 2}^{2}$ is independent of the random optical phase. To verify this phase diversity scheme in chirped lidar operation with homodyne detection, the outputs from both photodiodes were recorded by a digital sampling oscilloscope over a one-second time interval, producing the two independently faded power measurements shown in Fig. 10. The fading of these powers $P_{\mathrm{arm} 1}=I_{\mathrm{arm} 1}^{2}$ and $P_{\mathrm{arm} 2}=I_{\mathrm{arm} 2}^{2}$ varied between the lower limit of the noise floor and the upper limit of maximum carrier power. The power samples were then each normalized and summed, demonstrating in Fig. 10 that the combined power fading was significantly reduced. The residual power fluctuation of $<5 \mathrm{~dB}$ is attributed mainly to laser relative intensity noise (RIN) and the multi-path interference in the system.

Under a Doppler shift of the return signal, we expect a dualfrequency beat signal representing the separation of the signal and LO center frequencies. This should allow velocity measurement and determination of range through simple processing of the signal data.

\section{Free-Space LidAR Field Trials}

In the last section, to make comparisons between detection sensitivities of different system configurations and with the theoretical limit, an optical fiber delay line was used to simulate the target distance. This prevented ambiguities due to uncertainties in free-space coupling, air turbulence, and target reflectivity. In practical lidar altimeter applications, however, free-space optics has to be used which employs a telescope to collimate the optical signal onto the target. Therefore, a field trial using free-space optics is necessary to test the feasibility of the simplified coherent 


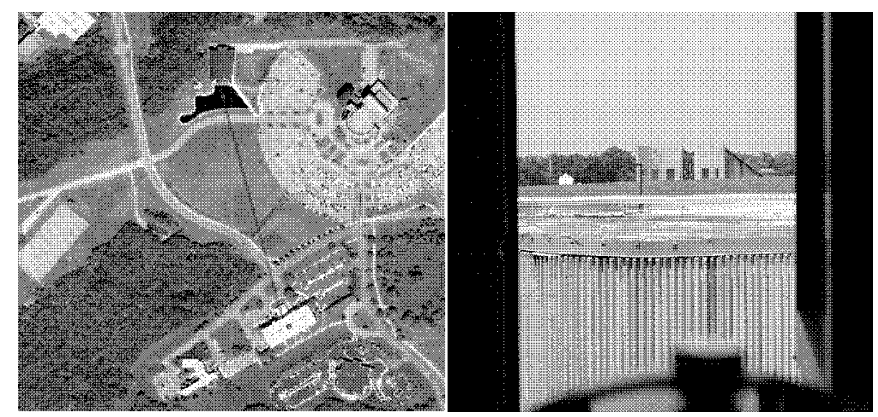

Fig. 11. Building target at 370-m range. Image from Google Earth (left), photo from lidar position (right).

homodyne detection in a lidar system. A 5-in-diameter commercial Newtonian telescope is used in this experiment which focuses the optical signal onto the target. The modulated optical signal is first split into two parts by a 10-dB fiber splitter. One part (10\%) goes through a praseodymium-doped fiber amplifier (PDFA) to boost the power for telescope, whereas the other part $(90 \%)$ is used as the LO for coherent homodyne detection. A polarization controller is used to adjust the polarization state of the $\mathrm{LO}$ and the $22.7-\mathrm{km}$ single-mode fiber delay line is still used to provide flexibility in adjusting the frequency of the de-chirped RF signal. A 3-dB fiber coupler is used to separate the transmitted signal and the detected signal from the telescope, and another 3-dB coupler is used to combine the optical signal with the LO. A balanced photodetector is used for homodyne detection and the de-chirped RF signal is measured by an RF spectrum analyzer. An APC (angled physical-contact) fiber connector was mounted to the telescope for transmission and reception to minimize the Fresnel reflection from the fiber terminal. The position of the fiber connector was adjusted to focus the transmitted beam at the distance of the target and in this way the reflected power from the target was thus reciprocally focused back onto the open fiber end.

In the first trial, a sheet of white paper was used as the target which was placed $50 \mathrm{~m}$ away from the telescope. In this measurement, the PDFA was not used because the optical power from the laser was high enough. The optical power reaching the target was found to be $-14 \mathrm{dBm}$ measured by a handheld power meter with the photodetector active area much larger than the beam size. Taking into account the telescope aperture radius $r$, spherical spreading of the reflected power at one way distance $R$, and the further loss $L_{3} \mathrm{~dB}$ due to the 3-dB fiber coupler, the total power loss is about $67 \mathrm{~dB}$ according to the relation

$$
\left(\frac{P_{\text {returned }}}{P_{\text {transmitted }}}\right)_{\mathrm{dB}}=10 \log _{10}\left(\frac{\pi r^{2}}{4 \pi R^{2}}\right)-L_{3 \mathrm{~dB}} .
$$

Using (10), the signal power returned to the optical receiver was estimated to be approximately $-81 \mathrm{dBm}$. The observed maximum SNR at this power level was about $12 \mathrm{~dB}$ although it fluctuated over time. The power fluctuation of the de-chirped signal is largely due to the well-known effect of carrier fading in homodyne detection, which can be overcome by using phase-diversity detection as discussed in the last section. This measured $-81 \mathrm{dBm}$ receiver sensitivity (with $12 \mathrm{~dB} \mathrm{SNR}$ ), as also shown

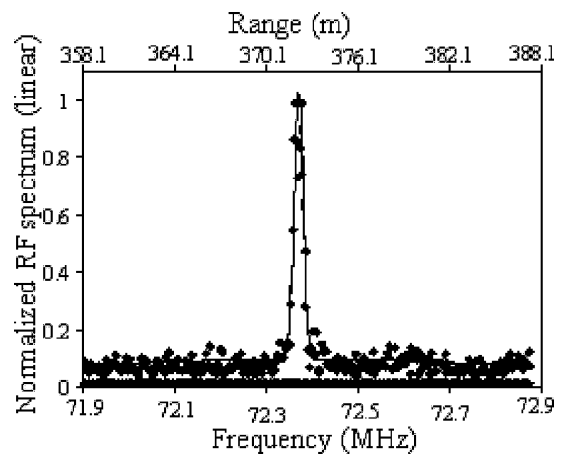

Fig. 12. Limestone target echo at $370 \mathrm{~m}$. (no averaging, 30-kHz res. BW, 500-ms sweep time over 2-MHz span).

in Fig. 7, is about $16 \mathrm{~dB}$ worse than the $-97 \mathrm{dBm}$ shot-noise limit. This discrepancy is mainly attributed to the coupling efficiency from the target to the single mode fiber. Obviously not all the optical power that enters the 5-in telescope can be captured by the fiber. Also, the reflectivity of the white paper target is expected to be less than $100 \%$. We believe this free-space detection sensitivity is promising yet still preliminary, and improved sensitivity may be realized by additional tuning of the optical system. Target speckle and free-space effects have not yet been included in the estimation of propagation loss, and these factors need to be included before the shot-noise limited sensitivity level can be determined.

A second trial was conducted using the limestone wall of a nearby building on the university campus as the target as shown in Fig. 11, and the distance was about $370 \mathrm{~m}$. Due to the increased distance, the PDFA was used to boost the transmitted optical power to approximately $8 \mathrm{dBm}$. Considering the useful gain of the PDFA was roughly $20 \mathrm{~dB}$, we estimate the power incident on the target was approximately $6 \mathrm{dBm}$. At this target distance it was still possible to detect the reflected lidar signal with approximately $12 \mathrm{~dB}$ SNR, again with significant fluctuation due to optical phase mismatch between the signal and the LO. Using the approximate distance of $370 \mathrm{~m}$ found with an aerial image as shown in Fig. 11 predicts a 72.3-MHz target beat frequency. The measured beat frequency was $72.38 \mathrm{MHz}$ indicating a true distance of $371.3 \mathrm{~m}$. Fig. 12 shows the beat signal captured from the spectrum analyzer.

\section{CONCLUSION}

We have demonstrated a simplified homodyne detection scheme for laser radar in which the LO is modulated by the same waveform as the optical signal, and de-chirping of the received FM pulse is accomplished in the optical domain. This system produces the observable radar echo directly at the photodetector output, and improves the detection sensitivity with shot-noise- limited performance. While kept at minimum hardware complexity, the system is capable of providing near quantum-limited detection sensitivity. In addition, linear chirping in the RF domain can be better controlled through digital signal processing in comparison to optical frequency chirping. Another major advantage of this arrangement is the reduction of required electrical bandwidth of the photodiode compared to conventional coherent detection schemes. In order 
to overcome signal fading problem common for all homodyne-based detection, we have demonstrated a phase diversity receiver using a $90^{\circ}$-hybrid optical coupler. Our free-space target detection experiments demonstrated a receiver sensitivity of approximately $-81 \mathrm{dBm}$, and successful detection of a limestone target at $370 \mathrm{~m}$.

\section{REFERENCES}

[1] C. Allen, Y. Cobanoglu, S. K. Chong, and S. Gogineni, "Performance of a $1319 \mathrm{~nm}$ laser radar using RF pulse compression," in Proc. 2001 Int. Geosci. Remote Sens. Symp. (IGARSS '01), Sydney, Australia, Jul. 2001, vol. Session SS52, pp. 997-999, Th03-04.

[2] D. F. Pierrottet, F. Amzajerdian, L. Petway, B. Barnes, G. Lockard, and M. Rubio, "Linear FMCW laser radar for precision range and vector velocity measurements," in Proc. Mater. Res. Soc. Symp., 2008, vol. 1076, 1076-K04-06.

[3] G. Keiser, Optical Fiber Communications, 3rd ed. New York: McGraw-Hill, 2000, ch. 6.

[4] P. Gatt and S. W. Henderson, "Laser radar detection statistics: A comparison of coherent and direct detection receivers," in Proc. SPIE, Apr. 2001, vol. 4377, pp. 251-262.

[5] A. Yariv, Optical Electronics. Geneva, IL: Holt, Rinehart and Winston, 1985 , pp. 362-375.

[6] R. Epworth, J. Whiteaway, and S. J. Savory, "3 Fibre I and Q Coupler," U.S. patent 6,859,586, Feb. 22, 2005.

[7] K. Yonenaga and S. Kuwano, "Dispersion-tolerant optical transmission system using duobinary transmitter and binary receiver," IEEE J. Lightw. Technol., vol. 15, no. 8, pp. 1530-1537, Aug. 1997.

[8] M. Schwartz, W. R. Bennett, and S. Stein, Communication Systems and Techniques. Piscataway, NJ: IEEE Press, 1996, ch. 3.
Peter Adany, received the B.S. and M.S. degrees in electrical engineering from the University of Kansas, Lawrence. He is currently working toward the Ph.D. degree in electrical engineering at the University of Kansas, where he is engaged in research using pulsed lasers and nonlinear fibers for bioimaging and other imaging applications.

His research interests include laser imaging, nonlinear optics, as well as signal processing. He has worked with the Center for Remote Sensing of Ice Sheets (CReSIS) to develop a high-performance laser radar for airborne ice field measurement missions.

Christopher Allen, photograph and biography not available at the time of publication.

Rongqing Hui received the B.S. degree in microwave communications in 1989 and the M.S. degree in lightwave technology in 1988, both from Beijing University of Posts and Telecommunications, Beijing, China, and the Ph.D. degree in electrical engineering from Politecnico di Torino, Turin, Italy, in 1993.

He joined Bell-Northern Research, Ottawa, ON, Canada, in 1994 as a Member of Scientific Staff, where he has worked in the research and development of high-speed optical transport networks. Since September 1997, he has been a faculty member in the Electrical Engineering and Computer Science Department, University of Kansas, where he is currently a Professor. He served as a Program Director at the U.S. National Science Foundation from 2006 to 2007, where he was in charge of the photonics and optoelectronics program. As an author or coauthor, he has published more than 90 technical papers in the area of fiber-optic communications and holds 13 U.S. patents.

Dr. Hui was a topic editor for the IEEE TRANSACTIONS ON COMMUNICATIONS from 2001 to 2006 and is currently serving as an Associate Editor of the IEEE JOURNAL OF QUANTUM ELECTRONICS. 